\title{
Osgood-Schlatter's Disease
}

National Cancer Institute

\section{Source}

National Cancer Institute. Osgood-Schlatter's Disease. NCI Thesaurus. Code C34874.

Osteochondrosis of the growth plate near the tibial tuberosity. 